\title{
Correlation between the characteristics of ultrasonic contrast and the regional distribution of tumor vascular heterogeneity in breast cancer
}

\author{
Xiaoling LENG ${ }^{1}$, Guofu HUANG², Fucheng MA ${ }^{1 \star}$ (D) , Jianbing DING ${ }^{3}$
}

\begin{abstract}
To clarify the correlation between qualitative and quantitative parameters of contrast-enhanced ultrasound and regional distribution differences of microvessel density (MVD) and vasculogenic mimic (VM) in breast cancer. 119 female patients with suspected breast cancer were involved. The clinicopathological parameters of patients were collected. Contrast-enhanced ultrasound was performed on patients. After the operation, according to the unified standard, the marginal zone, the central region and the paracancerous area of the breast cancer were sampled respectively. The MVD were labeled by immunohistochemistry with $\mathrm{CD} 34$, and tumor VM was labeled by CD34/PAS double staining. The distributions of MVD and VM in the marginal zone, the central area and the paracancerous area were decreased $(\mathrm{P}<0.05$, respectively), but the correlations between MVD, VM and contrast-enhanced ultrasound were not consistent. The quantitative parameters of contrast-enhanced ultrasound in marginal zone and central area were correlated with MVD. The positive rate of VM in marginal zone was also higher when breast cancer mass was larger and clinical stage was higher. When histological grade of breast cancer was high, the positive rates of VM in three regions were also high. The correlation between contrast-enhanced ultrasound and MVD was better than that of VM, but VM was associated with more prognostic factors than MVD.
\end{abstract}

Keywords: breast cancer; ultrasonic contrast; tumor vascular heterogeneity; regional distribution.

Practical Application: The combination of contrast-enhanced ultrasound and VM can not only reflect the growth of tumor blood vessels, but also predict the trend of tumor growth, metastasis and recurrence.

\section{Introduction}

Breast cancer (BC), one of the most common malignant tumors in women, is characterized by breast mass and the abnormal changes of nipple and areola in clinical (Lambertini et al., 2018). The occurrence and development of $\mathrm{BC}$ are a progressive process of multi-factor, multi-stage, multi-gene changes (Liu et al., 2009). BC is highly heterogeneous, and the prognosis of patients with the same clinical manifestations is very different. The prognostic factors determine the biological behavior of breast cancer, which further determine the histopathological changes (Zheng et al., 2019). The tumor microenvironment of breast cancer is composed of a variety of stromal cells, cytokines, chemokines and tumor stromal neovascularization, which is mainly manifested by malignant stroma and tumor vascular heterogeneity (Soysal et al., 2015).

Tumor vascular heterogeneity can be illustrated by the expression of microvessel density (MVD) and vasculogenic mimic (VM) (Saponaro et al., 2018). MVD refers to the number of microvessels per unit density in biological tissues such as skin, muscle, organ, etc. VM refers to that tumor cells mimic the angiogenesis of vascular endothelial cells and form cord like microcirculation channels through their own genotype reversal deformation and extracellular matrix remodeling. MVD is regarded as the "gold standard" to evaluate the angiogenesis of solid tumors. A large number of studies have shown that MVD is an independent prognostic factor for breast cancer (Laxmanan et al., 2005), which may be closely related to tumor invasion, metastasis, recurrence and prognosis. As another important index to describe the tumor vascular heterogeneity, VM plays an indispensable role in the occurrence, development and metastasis of breast cancer, although it is rare in breast cancer (Mitra et al., 2020).

Contrast-enhanced ultrasound uses contrast medium to increase the ultrasonic scattering of blood to improve the display of low velocity blood flow, which is of great value for the evaluation of tumor microenvironment and prognosis of breast cancer (BarZion et al., 2016). Contrast-enhanced ultrasound combined with biomarkers is a noninvasive, rapid and reproducible imaging method to evaluate the expression of tumor related genes/proteins, which can not only display the anatomical structure of tumor, but also evaluate the effect of tumor angiogenesis and anti-tumor angiogenesis and predict the prognosis (Forbrich et al., 2013; Warram et al., 2012; Jia et al., 2014; Liu et al., 2014; FernándezBarbero et al., 2020; Shabbir et al., 2020).

With the further understanding of tumor angiogenesis, the development of vascular functional molecular markers based on 
the tumor vascular heterogeneity has made it possible for tumor molecular imaging targeting tumor neovascularization. As a real contrast agent, the contrast-enhanced signal comes from the tumor vascular bed completely, which is more suitable for the research of tumor angiogenesis than other imaging examinations (Carrillo-Lopez et al., 2019; Piñon et al., 2019; Vidal et al., 2020). The purpose of this study was to explore the correlation between the perfusion characteristics of contrast-enhanced ultrasound and the regional distribution characteristics of MVD and VM in $\mathrm{BC}$, and to explore the value of contrast-enhanced ultrasound in the evaluation of angiogenesis and prognosis of breast cancer, so as to provide a reliable imaging basis for making a reasonable personalized treatment plan for patients with BC.

\section{Materials and methods}

\subsection{Patients}

This study was approved by the ethics committee of our hospital. Female patients with suspected breast cancer who were admitted to our hospital and finally operated on between January 2017 and October 2018 were enrolled in this study. A total of 119 patients with pathologically confirmed breast cancer were included in the study. All patients were informed of the possible condition and signed the informed consent.

Inclusion criteria: (1) Patients suspected of breast cancer in clinical and imaging studies and treated in this hospital; (2) No core needle biopsy, radiotherapy, chemotherapy and endocrinotherapy were performed before contrast-enhanced ultrasound and after operation; (3) Patients who were treated by operation, and the pathological results and lymph node metastasis were obtained; (4) The number of breast tumors was 1; (5) Patients who had complete clinical data, surgical and pathological data. Exclusion criteria: (1) Patients who were sensitive to contrast medium; (2) Patients who had hemopoietic insufficiency, and heart, liver and kidney insufficiencies; (3) Patients who had distant metastasis of breast cancer, primary focus of other parts, or breast metastatic cancer; (4) The multicentric lesions of one side of breast were beyond the scanning range of ordinary ultrasound probe, and it was difficult to ensure the reference property of breast tissue beside the lesions; (5) Patients who had bilateral breast cancer; (6) Patients with stage IV distant metastasis found by physical examination and related auxiliary examination (chest and abdomen CT, bone scan, etc.); (7) Patient who were unable to receive surgery due to their physical conditions and economic conditions; (8) Patients with breast cancer who had undergone breast mass puncture, chemotherapy, radiotherapy and endocrine therapy.

\subsection{Ultrasonic contrast of breast cancer}

SonoVue, an ultrasound contrast agent from Bracco, was used. Sulfur hexafluoride (SF6) (Bracco Suisse SA, Switzerland) with phospholipid microcapsules as contrast microbubbles. The average diameter of microbubbles was $2.5 \mu \mathrm{m}$. The microbubbles with diameter of less than $8 \mu \mathrm{m}$ were more than $90 \%$, which can safely pass through the pulmonary circulation and left ventricle, and finally be discharged through the lung. $\mathrm{pH}$ was 4.5-7.5. Before use, $5 \mathrm{~mL}$ of normal saline was injected and shook well to prepare microbubble suspension (concentration: $5 \mathrm{mg} / \mathrm{mL}$ ). In addition, the contrast mechanical index (MI) was 0.07 , and contrast agent ( $5 \mathrm{~mL} /$ person) was injected into the elbow vein. Dynamic contrast-enhanced ultrasound (CEUS) was performed, and then $5 \mathrm{~mL}$ normal saline was injected immediately after entering the timing state. The whole process of CEUS was recorded and saved, which is about $3 \mathrm{~min}$.

\subsection{Dyeing method of MVD count}

The marginal zone, central area and $2 \mathrm{~cm}$ adjacent to the carcinoma tissue from postoperative breast cancer specimen were stained by immunohistochemistry. In this study, streptomycin anti biotin protein - peroxidase conjugation (S-P) method was used, mouse anti human hematopoietic cell monoclonal antibody (CD34) (Abcam, USA) was used as the primary antibody to label vascular endothelial cells, Goat anti rabbit IgG antibody HRP polymer (abcam, USA) was used as the second antibody. The known positive tablets were used as the positive control, and Phosphate buffer solution (PBS) (Fuzhou Maixin Biotechnology Development Co., Ltd, Fuzhou, China) was used as the negative control instead of the first antibody.

The specific operation steps were as follows. After embedding in paraffin, the breast tissue was sectioned continuously with a thickness of $4 \mu \mathrm{M}$. Slices was baked overnight at $60^{\circ} \mathrm{C}$ followed by paraffin section dewaxed and hydrated. Then, slices were immersed in $3 \% \mathrm{H}_{2} \mathrm{O}_{2}$ (Tianjin Fuyu Fine Chemical Co., Ltd, Tianjin, China) for $15 \mathrm{~min}$ to eliminate endogenous peroxidase activity. After PBS washing, high temperature and high pressure method with citrate buffer $(\mathrm{pH}=6.0$ ) (Fuzhou Maixin Biotechnology Development Co., Ltd, Fuzhou, China) was used to repair tissue antigen. After PBS washing, the slices were incubated with primary antibody at $37^{\circ} \mathrm{C}$ for $1 \mathrm{~h}$ followed by incubating with second antibody at 37 ${ }^{\circ} \mathrm{C}$ for $30 \mathrm{~min}$. Finally, after staining with DAB chromogenic agent (Fuzhou Maixin Biotechnology Development Co., Ltd, Fuzhou, China), the nuclei were stained with $10 \%$ hematoxylin solution (Zhuhai beisuo Biotechnology Co., Ltd, Shenzhen, China) for 1 min and sealed with neutral resin (Shanghai specimen model factory, Shanghai, China).

\subsection{CD34 and PAS double staining method}

First, the sections were stained with CD34 (1:100 dilution) by immunohistochemistry. Vascular endothelial cells were stained with JJHDAB chromogenic agent and observed through optical microscope (OLYMPUS, Tokyo, Japan). The slices were then incubated with $0.5 \%$ periodic acid solution at room temperature for $10 \mathrm{~min}$, and incubated with Schiff reagent (eBioscience, USA) at $37^{\circ} \mathrm{C}$ for $20 \mathrm{~min}$ in dark. After dehydration and sealing in Leica automatic sealing machine, slices were observed under optical microscope. PBS, as a primary antibody, was a negative control, and the normal breast tissue stained cherry red was as a positive control in PAS staining.

\subsection{Observation on qualitative indexes of contrast-enhanced ultrasound}

Two experienced ultrasound doctors reviewed the stored images without knowing the clinical data of patients. Dynamic playback was 
used to observe the whole process of contrast-enhanced ultrasound. The blood flow morphological characteristics of contrast agent perfusion were analyzed by microvascular imaging. Qualitative indexes included enhancement shape (irregular or regular), enhancement boundary (unclear or clear), enhancement range (less than or equal to the two-dimensional ultrasound range, or greater than the two-dimensional ultrasound range), enhancement mode (speed up and speed down, speed up and slow down, slow up and speed down or slow up and slow down), enhancement intensity (non-high enhancement or high enhancement), distribution of contrast agent, sequence of contrast agent entering tumor (overall enhancement or centrifugal enhancement, or centripetal enhancement), whether there was perfusion defect (no, yes), and whether there was perforator (no, yes).

\subsection{Sampling method for quantitative parameter analysis of contrast-enhanced ultrasound}

Two experienced ultrasound doctors reviewed the stored images without knowing the clinical data of patients. Open the QLAB analysis software, avoid the nourishing blood vessels of the tumor, respectively, sample the most abundant area of blood flow imaging in the center and edge of the lesion and the breast tissue beside the lesion to obtain the regions of interest (ROI), and get the time intensity curve (TIC) of different areas.
Different regions of breast cancer were defined as shown in Figure 1. The observation contents included median intensity (MI), initial intensity (II), peak intensity (PI), rise time (RT), rising slope (RS) [(peak intensity - initial intensity) / (peak time - initial intensity)], descending slope (DS), time from peak to one half (DT/2), mean transit time (MTT), area under curve (AUC), and time to peak (TTP). After pre-experimental study, the two ultrasound doctors can achieve good consistency, and each analysis was required to achieve good consistency.

\subsection{MVD count and result judgment}

Two experienced pathologists counted MVD in three areas of all breast cancer lesions without knowing the clinicopathological information of the patients. The MVD counting standard referred to the previously reported method (Weidner, 1995). CD34 was mainly expressed in vascular endothelial cells. First, pathologists observed the whole section under low power microscope $(100 \times)$, and looked for three areas with the highest vascular density, namely "hot spots". Secondly, the number of vessels stained brownish yellow by CD34 was counted under high magnification $(200 \times)$. The mean value of MVD in three hot spots was taken as the MVD of this section (the areas of hemorrhage, necrosis, fibrosclerosis and marginal reaction in tumor tissue were not counted).

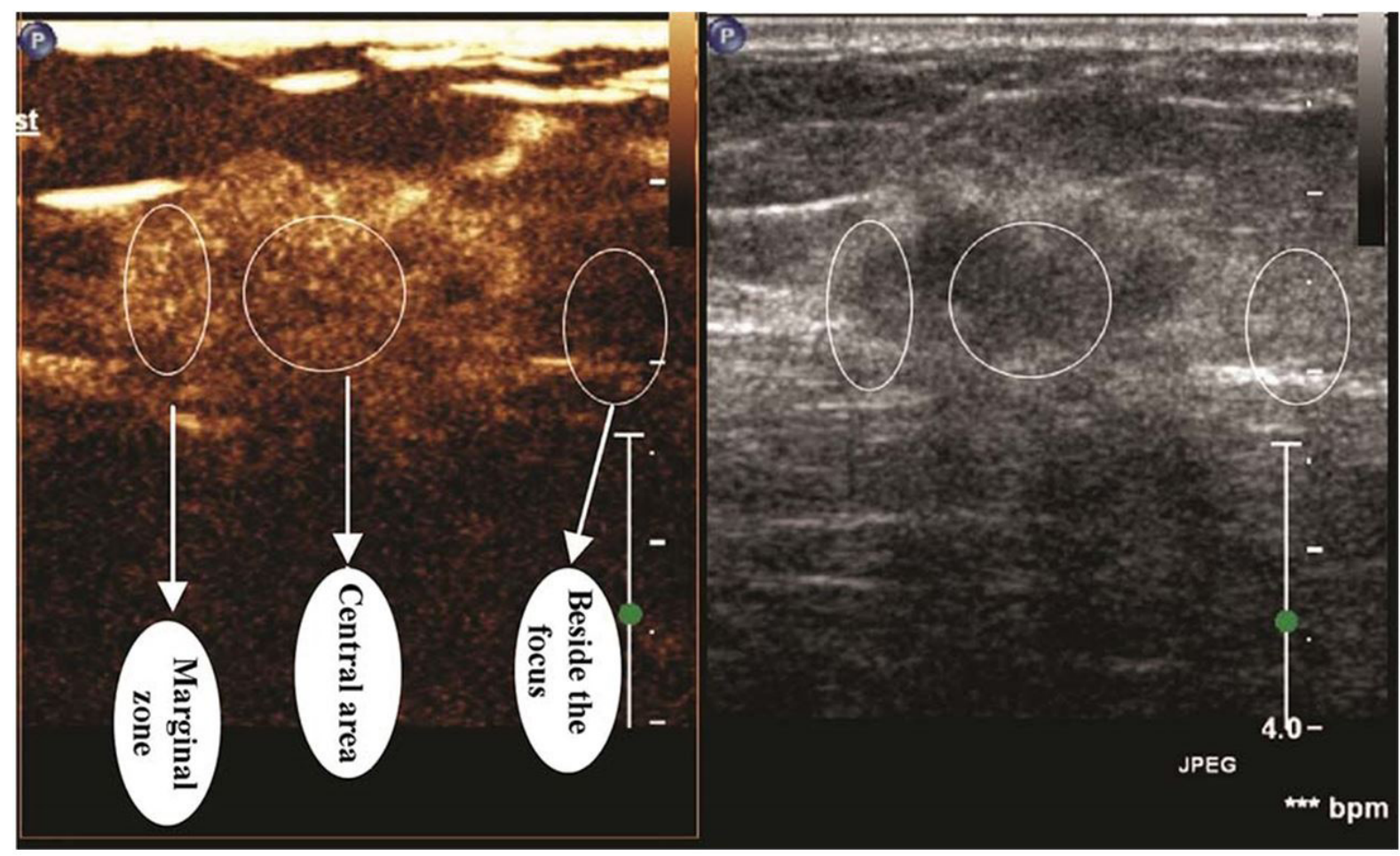

Figure 1. ROI sampling method for ultrasonic imaging. Central area: the area with a diameter of about $1 \mathrm{~cm}$ in the center of the focus. If the focus was small, the sampling frame can be appropriately reduced. Marginal zone: take the boundary of the enhancement range of the focus after contrast-enhanced ultrasound as the external area. In order to get as many marginal areas as possible, the sampling frame was oval. The sampling should avoid the tumor nutrient vessels as much as possible. A representative area should be selected. The ROI area in the marginal zone was similar to the ROI in the central area, and it should be on the same screen and depth as the sampling frame in the central area and the adjacent area of the focus. Breast tissue beside the focus: breast gland tissue was at the same depth and screen as the focus sampling frame. ROI sampling area was similar to the central area. Mark the study section of the mass with methylene blue, leave methylene blue in the sampling area of the marginal zone, and line the skin layer of the study section. 


\subsection{VM count and result judgment}

Two experienced pathologists counted VM in three areas of all breast cancer lesions without knowing the clinicopathological information of the patients. The inner wall of the duct was not lined with vascular endothelial cells. Instead, tumor cell cord which was formed with tumor cells generated by mimic the angiogenesis of the body and enclosed the duct. There are red blood cells in the tube, PAS positive substance around VM, no obvious necrosis and inflammatory cell infiltration around VM, which is a typical VM. If there is VM, it is positive; if there is no $\mathrm{VM}$, it is negative.

\subsection{Statistical analysis}

SPSS 22.0 was used as the statistical analysis software, and the significance test level was $\alpha=0.05$. The measurement data which conformed to the homogeneity of variance were expressed as $\bar{x} \pm s$, and which did not conform to were expressed as median and interquartile spacing. T-test and one-way ANOVA were used to analyze the data conforming to normal distribution, Kruskal Wallis test or Mann whiney test were used to analyze the data not conforming to normal distribution. $\chi^{2}$ test was used for counting data. Pearson correlation, Spearman correlation or variance analysis ETA coefficient were used for correlation analysis. $P<0.05$ was considered to be statistically significant.

\section{Results}

\subsection{Clinicopathological characteristics of patients with breast cancer}

A total of 119 patients with breast cancer participated in the study. Their average age was 51 years old (ranges from 29 to 81 years). Premenopausal patients accounted for $60.5 \%$ $(\mathrm{n}=72)$, postmenopausal patients accounted for $39.5 \%$ $(\mathrm{n}=47)$. All 119 cases were confirmed by pathology, including $10.1 \%(n=12)$ of ductal carcinoma in situ, $76.5 \%(n=91)$ of invasive ductal carcinoma and invasive lobular carcinoma, $13.4 \%(n=16)$ of invasive ductal carcinoma with carcinoma in situ and invasive lobular carcinoma with carcinoma in situ. All 119 specimens were histologically graded according to Scarff Bloom Richardson grading system, including 5 at grade 0,10 at grade I, 71 at grade II and 33 at grade III. All patients were examined for axillary lymphadenopathy, 29 of them showed metastasis. In $60.5 \%(n=72)$ patients, the tumor diameter was more than $2 \mathrm{~cm}$.

\subsection{Contrast analysis of contrast-enhanced ultrasound parameters and MVD in different regions of breast cancer}

The number of MVD in the marginal zone was greater than that in the central area, and the number of MVD in the central area was greater than that in the adjacent breast tissue $(P<0.05)$. The center zone refers to the center of the focus; the marginal zone refers to the edge of the focus. The contrast-enhanced ultrasound parameters, which were also different in the three regions of breast cancer, were MI, II and DS. The MI, II and DS in the marginal zone were larger than those in the central area, and the MI, II and DS in the central area were larger than those in the adjacent breast tissue $(P<0.05)$ (Table 1$)$. In addition, compared with the central area, the marginal zone had higher MVD, higher enhancement, more rapid retreat and lower perfusion in the early stage. However, the central area showed higher MVD, more rapid enhancement, higher perfusion, slower retreat and lower perfusion in the early stage in comparison with paracancerous area. Furthermore, there was significant difference in RS between the central area and the paracancerous areas, and there was marked difference in the TTP between the marginal zone and paracancerous areas (Figure 2).

MVD and some quantitative parameters of contrast-enhanced ultrasound were notably different in different regions of breast cancer. On this basis, we analyzed their correlation and the results were shown in Table 2. It showed that the MI, RS, II and DS in the marginal zone and central area were correlated with MVD, among which the MI and II were most correlated with MVD. In the paracancerous area, only the AS and DS were correlated with MVD. There was no correlation between TTP and MVD in different regions $(P>0.05)$.

\subsection{Contrast analysis of qualitative indexes of ultrasonic contrast and MVD in different regions of breast cancer}

We compared the qualitative indexes of contrast-enhanced ultrasound with MVD of different regions, and the results were shown in Table 3. $\mathrm{r}<0.4$ was considered as low correlation, $0.4 \leq \mathrm{r} \leq 0.7$ as moderate correlation, $\mathrm{r} \geq 0.7$ as high correlation.

Table 1. The regional distribution of MVD and quantitative parameters of contrast-enhanced ultrasound in breast cancer $\left(\mathrm{M}\left(\mathrm{P}_{25}, \mathrm{P}_{75}\right)\right)$.

\begin{tabular}{|c|c|c|c|c|c|}
\hline Indicators & Marginal zone & Central area & Paracancerous area & $\chi^{2}$ & $\mathbf{P}$ \\
\hline MVD & $49.51(18.00,73.00)^{\star \Delta}$ & $30.27(14.00,48.50)^{*}$ & $19.33(9.50,29.00)$ & 59.469 & $<0.001$ \\
\hline MI & $13.93(10.1,18.55)^{\star \Delta}$ & $7.99(5.95,10.47)^{\star}$ & $7.02(5.24,9.27)$ & 69.291 & $<0.001$ \\
\hline RS & $2.66(1.32,4.00)^{*}$ & $2.83(1.7,5.21)^{\star}$ & $1.75(0.92,3.27)$ & 47.970 & $<0.001$ \\
\hline II & $30.18(18.79,43.27)^{\star \Delta}$ & $39.32(28.33,56.65)^{*}$ & $45.45(28.29,66.88)$ & 149.748 & $<0.001$ \\
\hline DS & $19.67(16.14,24.61)^{* \Delta}$ & $18.81(15.3,26.61)^{\star}$ & $21.92(16.93,34.45)$ & 79.023 & $<0.001$ \\
\hline TTP & $9.58(5.81,12.72)^{\star}$ & $5.82(3.34,7.76)$ & $5.19(2.59,6.92)$ & 6.175 & 0.046 \\
\hline
\end{tabular}

${ }^{\star} \mathrm{P}<0.05$ : marginal zone or central area vs. paracancerous area; ${ }^{\triangle} \mathrm{P}<0.05$ : marginal zone or paracancerous area vs. central area. MVD: microvessel density; MI: median intensity; RS rising slope; II: initial intensity; DS: descending slope; TTP: time to peak. 


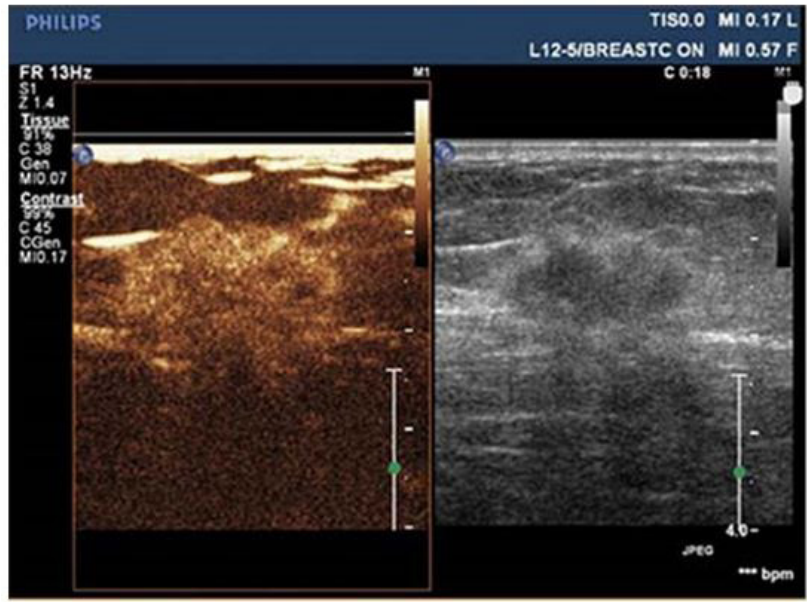

A

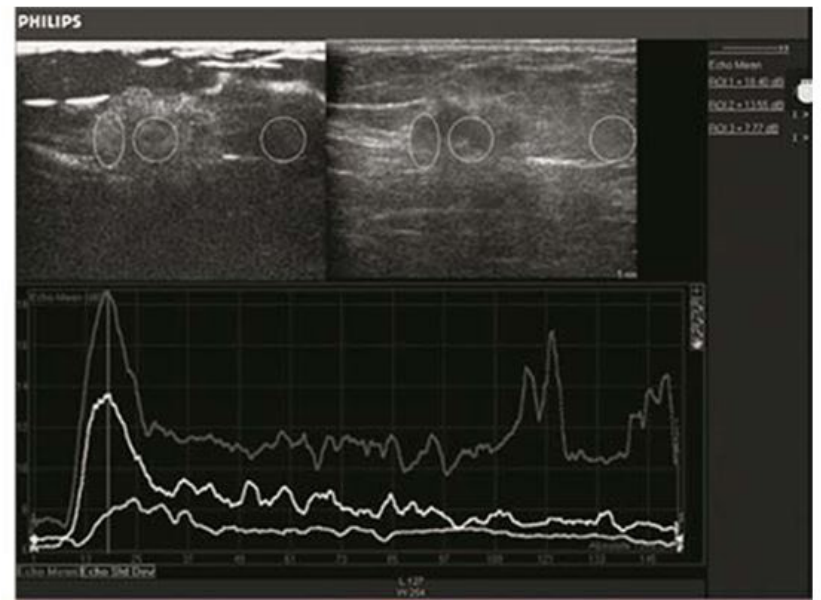

B
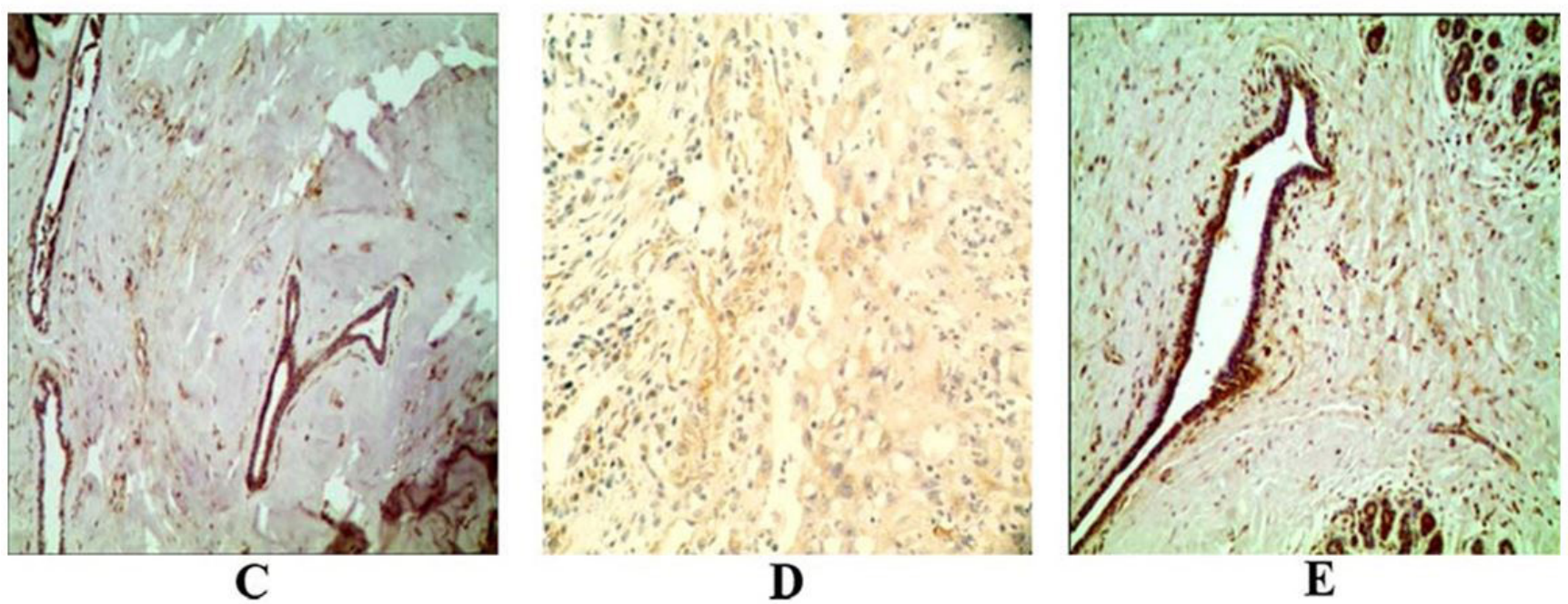

Figure 2. The differences of perfusion characteristics of ultrasound and MVD in different regions of breast cancer. (A) Ultrasonic contrast in patients with breast cancer; (B) TIC curve in different regions of breast cancer; (C) MVD in marginal zone of breast cancer ( $\times 100$ times); (D) MVD in central area of breast cancer ( $\times 100$ times); (E) MVD in paracancerous area of breast cancer $(\times 100$ times).

Table 2. Correlation between MVD and quantitative parameters of contrast-enhanced ultrasound in different regions of breast cancer.

\begin{tabular}{|c|c|c|c|c|c|c|c|c|c|c|}
\hline \multirow{2}{*}{ MVD } & \multicolumn{2}{|c|}{ MI } & \multicolumn{2}{|c|}{ RS } & \multicolumn{2}{|c|}{ II } & \multicolumn{2}{|c|}{ DS } & \multicolumn{2}{|c|}{ TTP } \\
\hline & $\mathbf{r}$ & $\mathbf{P}$ & $\mathbf{r}$ & $\mathbf{P}$ & $\mathbf{r}$ & $\mathbf{P}$ & $\mathbf{r}$ & $\mathbf{P}$ & $\mathbf{r}$ & $\mathbf{P}$ \\
\hline Marginal zone & 0.524 & $<0.001^{*}$ & 0.227 & $0.013^{*}$ & 0.407 & $<0.001^{\star}$ & 0.291 & $0.001^{\star}$ & -0.004 & 0.966 \\
\hline Central area & 0.411 & $<0.001^{\star}$ & 0.182 & $0.047^{\star}$ & 0.427 & $<0.001^{*}$ & 0.305 & $0.001^{*}$ & -0.137 & 0.137 \\
\hline Paracancerous area & 0.134 & 0.146 & 0.214 & $0.02^{*}$ & 0.072 & 0.437 & 0.182 & $0.047^{\star}$ & -0.027 & 0.773 \\
\hline
\end{tabular}

${ }^{*} \mathrm{P}<0.05$.

The results showed that there was a moderate correlation between the filling defect and MVD in the marginal zone, a high correlation between the filling defect and MVD in the central area, and no correlation between the filling defect and MVD in the paracancerous area. The enhancement degree was highly correlated with MVD in the three areas. However, there was no correlation between the enhanced range of lesions and MVD in the three areas. Figure 3 showed the breast cancer with filling defect after ultrasonic contrast, of which the MVD in the central area was lower than that without filling defect.

In addition, the results showed that there was no significant difference in MVD in the same region between breast cancer with 
focus enhancement range greater than two-dimensional (2-D) ultrasound and breast cancer with focus enhancement range equal to $2 \mathrm{D}$ ultrasound $(P>0.05)$. Compared with breast cancer without filling defect, the MVD of marginal zone was larger, and the MVD of central area was larger in breast cancer with filling defect $(P<0.05)$. However, there was no significant difference in MVD of paracancerous areas $(P>0.05)$. Compared with the none high enhanced breast cancer, the MVD of each region in high enhanced breast cancer were higher $(\mathrm{P}<0.05$, respectively) (Table 4$)$.

\subsection{Correlation between the qualitative indexes of ultrasonic contrast and VM in breast cancer}

The results showed that the positive rates of VM in the marginal zone and central area of breast cancer with filling defect were

Table 3. Correlation between MVD in three different regions of breast cancer and qualitative characteristics of contrast-enhanced ultrasound.

\begin{tabular}{|c|c|c|c|c|c|c|}
\hline \multirow{2}{*}{ Indicators } & \multicolumn{2}{|c|}{ MVD in marginal zone } & \multicolumn{2}{|c|}{ MVD in central area } & \multicolumn{2}{|c|}{ MVD in paracancerous area } \\
\hline & $\mathbf{r}$ & $\mathbf{P}$ & $\mathbf{r}$ & $\mathbf{P}$ & $\mathbf{r}$ & $\mathbf{P}$ \\
\hline Filling defect & 0.632 & $0.012^{*}$ & 0.787 & $<0.001^{*}$ & 0.490 & 0.251 \\
\hline Enhancement degree & 0.868 & $<0.001^{\star}$ & 0.891 & $<0.001^{*}$ & 0.753 & $<0.001^{*}$ \\
\hline Scope of mass after angiography & 0.550 & 0.234 & 0.495 & 0.804 & 0.515 & 0.198 \\
\hline
\end{tabular}

${ }^{*} \mathrm{P}<0.05$.

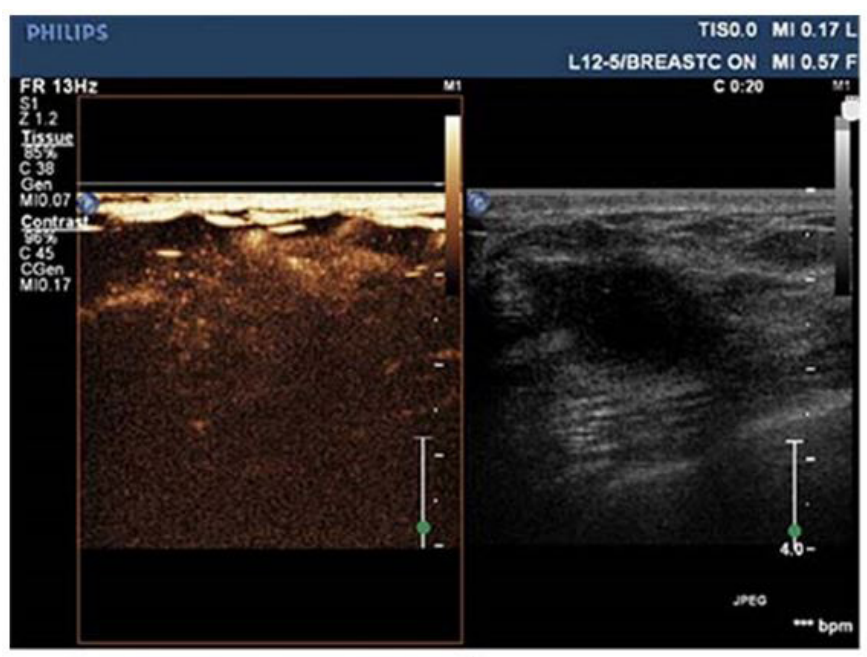

A

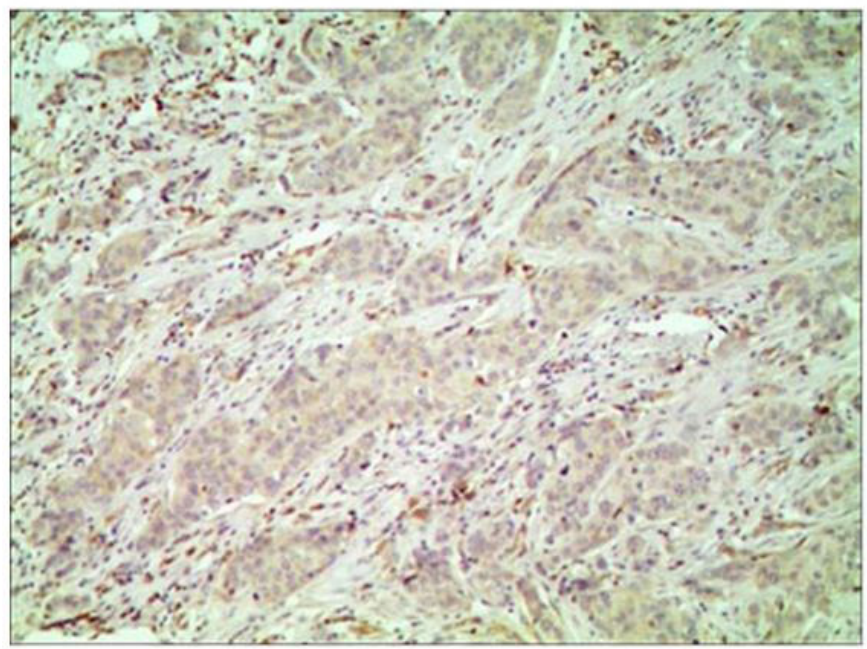

C

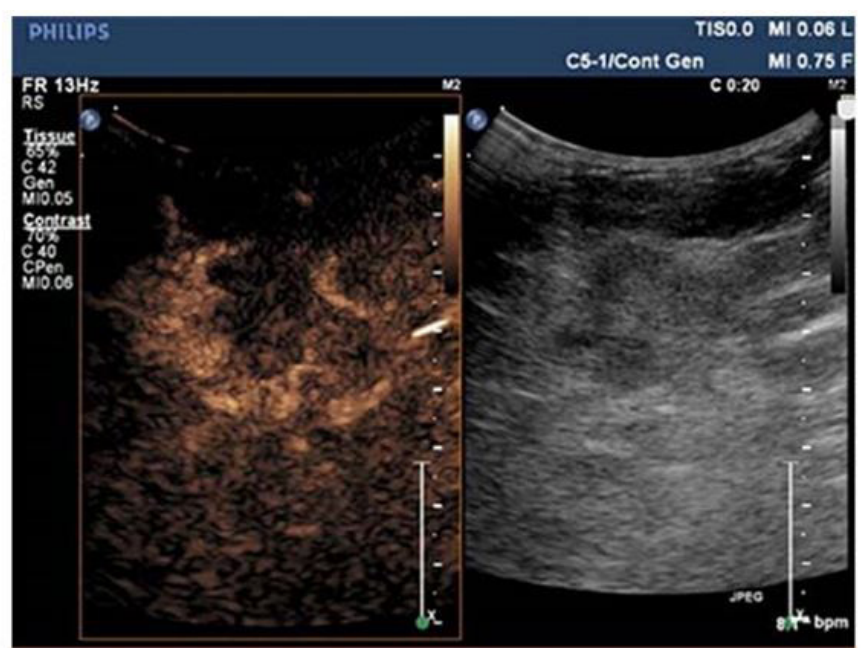

B

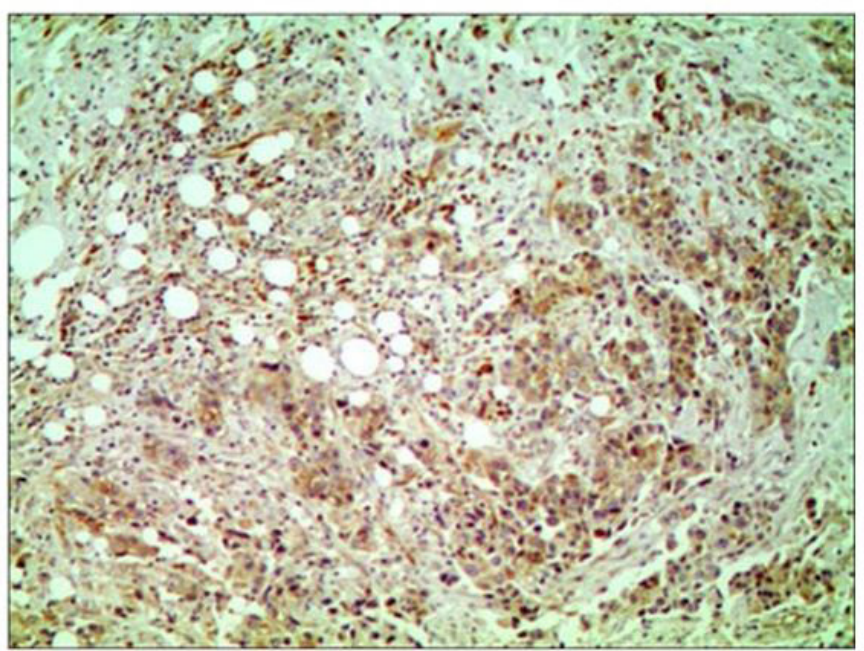

D

Figure 3. Difference between MVD and filling defect in ultrasonic contrast. (A) No filling defect after contrast-enhanced ultrasound; (B) Filling defect after contrast-enhanced ultrasound; (C) MVD in central area of no filling defect ( $\times 100$ times); (D) MVD in central area of filling defect $(\times 100$ times). 
higher than that in the marginal zone and central area of breast cancer without filling defect $(P<0.001$, respectively). However, there was no marked difference in those in paracancerous area of breast cancer $(P>0.05)$. Furthermore, there were no differences in the positive rates of VM in the marginal zone, the central area and the paracancerous area between breast cancer with and without high enhancement $(P>0.05)$. The positive rate of VM in the marginal zone of breast cancer with mass range larger than that of 2-D ultrasound was higher than that of breast cancer with mass range equal to that of $2-\mathrm{D}$ ultrasound $(P<0.05)$. However, there were no differences in the positive rates of VM in the central area and the paracancerous area $(P>0.05$, respectively) (Table 5).

\subsection{Correlation between quantitative parameters of ultrasonic contrast and VM in different regions of breast cancer}

We analyzed the correlation between quantitative parameters of ultrasonic contrast and VM in different regions of breast cancer. The results showed that there were no significant differences in these parameters, MI, RS, II, DS and TTP, in the three areas between VM negative breast cancer and VM positive breast cancer $(P>0.05$, respectively) (Table 6).

\subsection{The relevance between MVD and VM of breast cancer and lesion size, histological grade and clinical stage}

It showed that the differences of MVD in marginal zone and central area were statistically significant between breast cancer with different histological grades $(P<0.05)$. That is to say, the higher the histological grade, the higher the MVD in the marginal and central area of breast cancer. However, the different histological grades of breast cancer were not related to MVD in the paracancerous area $(P>0.05)$. However, there was no significant differences in MVD in the three areas between breast cancer with different lesion size and clinical stage $(P>0.05)$ (Table 7$)$.

The results showed that the positive rate of VM in the marginal zone of breast cancer more than $2 \mathrm{~cm}$ was significantly higher than that in breast cancer less than $2 \mathrm{~cm}(\mathrm{P}<0.001)$, but there were no significant differences in the positive rate of VM in the central area and paracancerous area $(P>0.05)$. Furthermore, for breast cancer with higher histological grade, the positive rates of VM in the three areas were significantly higher than those in breast cancer with lower histological grade $(P<0.05)$. The positive rate of VM in the marginal zone of breast cancer with clinical stage more than 1 was higher than that in stage 1 breast cancer $(P<0.05)$, but there was no significant difference in the positive rates of $\mathrm{VM}$ in the central area and the paracancerous area between breast cancer with clinical stage more than 1 and stage 1 breast cancer $(P>0.05)$ (Table 8$)$.

\section{Discussion}

Neovascularization plays an important role in the occurrence, development, invasion and metastasis of tumor, which significantly affects the biological behavior and prognosis of tumor (Dineshkumar et al., 2015). At present, there is no direct

Table 4. Distribution difference of MVD in a certain area in ultrasound contrast characteristics of breast cancer $((\bar{x} \pm S) / \mathrm{HP})$.

\begin{tabular}{|c|c|c|c|c|c|c|c|c|c|c|c|c|}
\hline \multirow{2}{*}{ Areas } & \multicolumn{2}{|c|}{ Enhancement range } & & \multirow{2}{*}{$\mathbf{p}$} & \multicolumn{2}{|c|}{ Filling defect } & & \multirow{2}{*}{$\mathbf{P}$} & \multicolumn{2}{|c|}{ Enhancement degree } & & \multirow{2}{*}{$\mathbf{P}$} \\
\hline & $>$ & $=$ & & & Yes & no & & & high & None high & & \\
\hline Marginal zone & $50.87 \pm 13.34$ & $48.54 \pm 15.91$ & 1.224 & 0.222 & $50.59 \pm 12.07$ & $47.78 \pm 11.52$ & 3.883 & $0.047^{*}$ & $53.52 \pm 11.18$ & $46.48 \pm 11.61$ & 4.765 & $<0.001^{*}$ \\
\hline Central area & $31.35 \pm 12.10$ & $28.46 \pm 13.58$ & 1.733 & 0.084 & $24.68 \pm 5.51$ & $36.14 \pm 13.36$ & 8.650 & $<0.001^{*}$ & $38.81 \pm 12.60$ & $22.68 \pm 8.52$ & 11.568 & $<0.001^{\star}$ \\
\hline $\begin{array}{l}\text { Paracancerous } \\
\text { area }\end{array}$ & $20.55 \pm 4.18$ & $19.32 \pm 6.56$ & 1.725 & 0.086 & $19.85 \pm 3.79$ & $18.91 \pm 4.35$ & 1.777 & 0.076 & $23.90 \pm 4.08$ & $17.50 \pm 3.08$ & 13.657 & $<0.001^{\star}$ \\
\hline
\end{tabular}

${ }^{\star} \mathrm{P}<0.05$.

Table 5. The distribution difference of VM positive rate in a region of breast cancer in qualitative index of ultrasound contrast imaging (n\%).

\begin{tabular}{|c|c|c|c|c|c|c|c|c|c|c|c|c|}
\hline \multirow{2}{*}{ Indicators } & \multicolumn{2}{|c|}{ Marginal zone } & \multirow{2}{*}{$\chi^{2}$} & \multirow{2}{*}{$\mathbf{P}$} & \multicolumn{2}{|c|}{ Central area } & \multirow{2}{*}{$\chi^{2}$} & \multirow{2}{*}{$\mathbf{P}$} & \multicolumn{2}{|c|}{ Paracancerous area } & \multirow{2}{*}{$\chi^{2}$} & \multirow{2}{*}{$\mathbf{P}$} \\
\hline & + & - & & & + & - & & & + & - & & \\
\hline Filling defect & & & 16.965 & $0.001^{*}$ & & & & & & & & \\
\hline No & $38(46.34)$ & $44(53.66)$ & & & $35(42.68)$ & $47(57.32)$ & & & $6(7.32)$ & $76(92.68)$ & & \\
\hline Enhancement degree & & & 0.274 & 0.673 & & & & & & & & \\
\hline None-high & $17(54.84)$ & $14(45.16)$ & & & $9(29.03)$ & $22(70.97)$ & & & $0(0.00)$ & $31(100.00)$ & & \\
\hline Enhancement range & & & 5.776 & $0.016^{*}$ & & & & & & & & \\
\hline$>$ & $59(64.84)$ & $32(35.16)$ & & & $29(31.87)$ & $62(68.13)$ & 0.53 & 0.467 & $9(9.89)$ & $82(90.11)$ & 0.084 & 0.772 \\
\hline$=$ & $11(39.29)$ & $17(60.71)$ & & & $11(39.29)$ & $17(60.71)$ & & & $2(7.14)$ & $26(92.86)$ & & \\
\hline
\end{tabular}

${ }^{\star} \mathrm{P}<0.05$. 
Table 6. The differences of the contrast-enhanced ultrasound parameters in the three areas between VM negative and VM positive $\left(M\left(P_{25}, P_{75}\right)\right)$.

\begin{tabular}{|c|c|c|c|c|c|c|c|}
\hline Areas & VM & Cases & MI & RS & II & DS & TTP \\
\hline \multirow[t]{4}{*}{ Marginal zone } & VM positive & 70 & $11.91(7.10,17.95)$ & $2.53(1.68,3.55)$ & $7.91(5.85,11.68)$ & $1.84(1.07,2.30)$ & $18.55(14.83,21.61)$ \\
\hline & VM negative & 49 & $12.02(7.86,14.97)$ & $2.49(1.62,3.5)$ & $8.70(4.16,12.61)$ & $1.78(1.18,2.73)$ & $19.75(16.39,24.61)$ \\
\hline & $\mathrm{Z}$ & & -0.103 & -0.305 & -0.116 & -0.287 & -1.293 \\
\hline & $\mathrm{P}$ & & 0.918 & 0.760 & 0.908 & 0.774 & 0.196 \\
\hline \multirow[t]{4}{*}{ Central area } & VM positive & 40 & $6.97(4.88,8.79)$ & $1.42(0.85,2.36)$ & $2.88(1.96,4.74)$ & $1.00(0.70,1.51)$ & $18.88(15.62,24.16)$ \\
\hline & VM negative & 79 & $6.22(4.25,8.71)$ & $1.58(0.64,2.72)$ & $2.73(1.64,5.18)$ & $1.00(0.43,1.80)$ & $18.71(14.80,27.54)$ \\
\hline & $\mathrm{Z}$ & & -0.503 & -0.335 & -0.366 & -0.096 & -0.315 \\
\hline & $\mathrm{P}$ & & 0.615 & 0.738 & 0.715 & 0.924 & 0.753 \\
\hline \multirow{4}{*}{$\begin{array}{c}\text { Paracancerous } \\
\text { area }\end{array}$} & VM positive & 11 & $6.32(4.12,8.90)$ & $1.30(1.05,2.61)$ & $1.48(0.43,2.73)$ & $0.84(0.31,1.00)$ & $20.16(13.48,25.31)$ \\
\hline & VM negative & 108 & $5.34(2.66,7.38)$ & $1.07(0.58,2.19)$ & $1.61(0.77,2.78)$ & $0.98(0.23,0.99)$ & $21.38(13.48,25.31)$ \\
\hline & $\mathrm{Z}$ & & -1.212 & -1.087 & -0.091 & -0.343 & -0.436 \\
\hline & $\mathrm{P}$ & & 0.226 & 0.277 & 0.927 & 0.732 & 0.663 \\
\hline
\end{tabular}

VM: vasculogenic mimic. MI: median intensity.

Table 7. The relevance between the distribution of MVD and clinical pathological factors in different breast cancer regions.

\begin{tabular}{|c|c|c|c|c|c|c|}
\hline \multirow{2}{*}{ Factors } & \multicolumn{2}{|c|}{ Marginal zone } & \multicolumn{2}{|c|}{ Central area } & \multicolumn{2}{|c|}{ Paracancerous area } \\
\hline & $\mathrm{Z} / \chi^{2}$ & $\mathbf{P}$ & $\mathrm{Z} / \chi^{2}$ & $\mathbf{P}$ & $\mathrm{Z} / \chi^{2}$ & $\mathbf{P}$ \\
\hline Lesion size & 2.91 & 0.233 & -0.26 & 0.794 & -0.54 & 0.591 \\
\hline Histological grade & -2.28 & $0.022^{*}$ & -1.68 & $0.047^{\star}$ & 3.43 & 0.180 \\
\hline Clinical stage & -1.91 & 0.056 & -0.44 & 0.661 & -0.22 & 0.826 \\
\hline
\end{tabular}

${ }^{\star} \mathrm{P}<0.05$.

Table 8. The distribution difference of VM positive rate in a region of breast cancer in prognostic factors (n\%).

\begin{tabular}{|c|c|c|c|c|c|c|c|c|c|c|c|c|}
\hline \multirow{2}{*}{ Indicators } & \multicolumn{2}{|c|}{ Marginal zone } & \multirow{2}{*}{$\chi^{2}$} & \multirow{2}{*}{$\mathbf{P}$} & \multicolumn{2}{|c|}{ Central area } & \multirow{2}{*}{$\chi^{2}$} & \multirow{2}{*}{$\mathbf{P}$} & \multicolumn{2}{|c|}{ Paracancerous area } & \multirow{2}{*}{$\chi^{2}$} & \multirow{2}{*}{$\mathbf{P}$} \\
\hline & + & - & & & + & - & & & + & - & & \\
\hline \multicolumn{13}{|l|}{ Lesion size } \\
\hline$>2 \mathrm{~cm}$ & $53(73.61)$ & $19(40.28)$ & & & $27(37.50)$ & $45(62.50)$ & & & $10(13.89)$ & $62(86.11)$ & & \\
\hline \multicolumn{13}{|c|}{ Histological grade } \\
\hline grade II & $38(53.52)$ & $33(45.83)$ & & & $24(33.80)$ & $47(66.20)$ & & & $2(2.82)$ & $69(97.18)$ & & \\
\hline grade III & $30(90.91)$ & $3(9.09)$ & & & $15(45.45)$ & $18(54.55)$ & & & $9(27.27)$ & $24(72.73)$ & & \\
\hline Clinical stage & & & 10.806 & $0.001^{*}$ & & & 0.590 & 0.442 & & & 1.624 & 0.203 \\
\hline$<$ stage I & $11(34.37)$ & $21(65.63)$ & & & $9(28.12)$ & $23(71.88)$ & & & $1(3.13)$ & $31(96.87)$ & & \\
\hline
\end{tabular}

${ }^{*} \mathrm{P}<0.05$.

and effective method to detect the angiogenesis of tumor. MVD as an indicator of angiogenesis activity has been proposed and verified in many tumor studies (Lin et al., 2011; Korn et al., 2014), which is a more objective standard to evaluate the degree of angiogenesis in malignant tumors. Through a large number of clinical tests, some scholars have found that the microvessel density in tumor tissue is significantly higher than that in normal tissue around the tumor (Sharma et al., 2011). The more microvessels in tumor tissue, the greater the chance of tumor cells entering the blood circulation, and the more likely it is to increase the occurrence and metastasis of tumor.

This study showed that there were significant differences in MVD expression among the marginal zone, central area and paracancerous area, and MVD rich area was located around the cancer focus. The analysis of contrast-enhanced ultrasound also showed that the differences of MI, II and DS in different regions 
of breast cancer were consistent with the difference of MVD, that was, there was a difference of blood perfusion in different regions of breast cancer. Compared with the central region of breast cancer, the marginal zone of breast cancer had a higher tumor vascular density, a high enhancement, a slow retreat and a lower level of perfusion in the early stage of enhancement. The reason was that the vascular heterogeneity in the marginal area of breast cancer was more obvious, and the defect of tumor blood vessel wall structure was obvious, which led to the increase of vascular permeability, interstitial edema, and obstruction of tissue space reflux. As a result, the descending branch of the time intensity curve was more gentle than the central region, and the perfusion intensity was higher, but the initial intensity was lower at the initial stage of perfusion, which may be related to the resistance of microbubble filling at the initial stage.

In addition, the results confirmed that the marginal zone showed a higher perfusion trend than the central area, suggesting that the immunohistochemical MVD count was consistent with the contrast findings, and the degree of vascularization of the Marginal zone was higher than that of the central area. It was confirmed that the distribution of new microvessels in tumor tissue was not uniform, which was mainly distributed on the edge of tumor active growth. In this study, the correlation analysis further confirmed that the perfusion parameters, MI, II, DS, RS and TTP, were almost related to MVD in the central area, regardless of the marginal zone or the central area, among which, the MI and II were significantly related, which again proved the consistency of tumor vascular heterogeneity in pathological morphology and hemodynamics.

At present, three ways of blood supply have been found, namely, classic endothelium-dependent blood vessels, mosaic blood vessels and angiogenic mimicry (Chen et al., 2009). However, the traditional anti angiogenic treatment is mainly aimed at the classic blood vessels, and the formation of blood vessels by tumor itself has not been considered. The concept of VM enriches and perfects the theory of tumor blood supply. VM is a pipe line system that can transport blood by changing the shape of tumor cells and promoting the production of extracellular matrix to imitate the structure of classic endothelial vessel wall, so as to remodel and perfect the microcirculation of tumor, and connect with the classic endothelial vessel of the host, so that the tumor can obtain enough blood supply to play Its potential of infinite proliferation (Mao et al., 2015; Chen \& Chen, 2014; Fan \& Sun, 2010). Due to the small number of VM, this study, like most of the literature, explored the difference of the positive rates $\mathrm{v}$, and does not carry out the same quantitative statistics as MVD. But its existence is closely related to the biological behavior of tumor. This study confirmed that the positive rates of VM in the marginal zone and central area of breast cancer with filling defect after ultrasonic contrast would be higher. It was worth noting that regardless of the perfusion degree of contrast-enhanced ultrasound, the positive rate of VM in each region was not different. This result was greatly different from the previous MVD analysis in our study, which also showed that the breast cancer area with VM did not necessarily show high-intensity perfusion, and did not necessarily exist in a large number of tumor vessels. The positive rate of VM was consistent with the distribution of MVD in the three regions of breast cancer.
Furthermore, our results showed that there was no significant correlation between the positive rate of VM in each region of breast cancer and the parameters, MI, II, DS, RS and TTP. The correlation analysis of qualitative and quantitative parameters of contrast-enhanced ultrasound showed that these parameters and qualitative indexes were related to contrast-enhanced degree and contrast-enhanced mode, so the correlation of VM and quantitative parameters more objectively reflected that the positive rate of $\mathrm{VM}$ was not related to contrast-enhanced degree, and again highlighted that the correlation between VM and MVD and contrast-enhanced ultrasound was different. MVD, as a quantitative index of tumor blood vessels widely existing in the focus, can reflect the blood supply state of the tumor in theory. However, there is a small amount of VM in the tumor. For qualitative analysis, there is no common change trend with the number of tumor blood vessels, so VM cannot be associated with most quantitative parameters of contrast-enhanced ultrasound.

Most studies showed that angiogenesis of breast cancer was not necessarily related to tumor size and clinical stage, but closely related to histological grading (Onji et al., 2012). The higher the histological grade, the greater the malignant degree, the stronger the ability of angiogenesis, and the worse the prognosis. The results of this study confirmed that the positive rate of VM correlated more prognostic indicators than MVD, which showed the clinical value of evaluating the prognosis of breast cancer. However, the correlation between VM and contrast-enhanced ultrasound was less than MVD, especially the correlation between VM and quantitative parameters of time intensity curve was worse than MVD. Therefore, it was believed that contrast-enhanced ultrasound had a good correlation with MVD, but the positive rate of VM was better than MVD count in evaluating the prognosis.

\section{Conclusions}

In conclusion, our results showed that the distributions of MVD and VM in the marginal zone, central area and paracancerous area were consistent, showing a decreasing distribution, but the distributions of MVD and VM in breast cancer were not consistent with contrast-enhanced ultrasound. The correlation between contrast-enhanced ultrasound and MVD was significantly better than the correlation between contrast-enhanced ultrasound and VM, but VM had more prognostic indicators than MVD. The combination of contrast-enhanced ultrasound and VM can not only reflect the growth of tumor blood vessels, but also predict the trend of tumor growth, metastasis and recurrence.

\section{Acknowledgements}

This work was supported by the National Natural Science Foundation of China (No.81660496) and Xinjiang Youth Science and Technology Innovation Talent Training Project (No.2017Q075).

\section{References}

Bar-Zion, A., Yin, M., Adam, D., \& Foster, F. S. (2016). Functional flow patterns and static blood pooling in tumors revealed by combined contrast-enhanced ultrasound and photoacoustic imaging. Cancer 
Research, 76(15), 4320-4331. http://dx.doi.org/10.1158/0008-5472. CAN-16-0376. PMid:27325651.

Carrillo-Lopez, L. M., Luna-Rodriguez, L., Alarcon-Rojo, A. D., \& Huerta-Jimenez, M.. (2019). High intensity ultrasound homogenizes and improves quality of beef longissimus dorsi. Food Sci. Technol, 39(Suppl. 1), 332-340. http://dx.doi.org/10.1590/fst.05218.

Chen, L. X., Sun, B. C., Zhang, S. W., He, Y. J., Li, X. R., \& He, Z. J. (2009). The mechanisms of microenvironments influence on vasculogenic mimicry between introcular and subcutaneous melanoma. Chinese Journal of Ophthalmology, 45(7), 641-646. PMid:19957694.

Chen, Y. S., \& Chen, Z. P. (2014). Vasculogenic mimicry: a novel target for glioma therapy. Chinese Journal of Cancer, 33(2), 74-79. http://dx.doi.org/10.5732/cjc.012.10292. PMid:23816560.

Dineshkumar, T., Priyadharsini, N., Gnanaselvi, U. P., Sathishkumar, S., Srikanth, R. P., \& Nagarathinam, A. E. (2015). Evaluation and comparison of vascular endothelial growth factor expression between ameloblastoma and keratocystic odontogenic tumor. Journal of International Oral Health, 7(1), 48-52. PMid:25709368.

Fan, Y. Z., \& Sun, W. (2010). Molecular regulation of vasculogenic mimicry in tumors and potential tumor-target therapy. World Journal of Gastrointestinal Surgery, 2(4), 117-127. http://dx.doi.org/10.4240/ wjgs.v2.i4.117. PMid:21160860.

Fernández-Barbero, G., Pinedo, C., Espada-Bellido, E., FerreiroGonzález, M., Carrera, C., Palma, M., \& García-Barroso, C. (2020). Optimization of ultrasound-assisted extraction of bioactive compounds from jabuticaba (Myrciaria cauliflora) fruit through a Box-Behnken experimental design. Food Science and Technology, 39(4), 1018-1029. http://dx.doi.org/10.1590/fst.16918.

Forbrich, A., Paproski, R., Hitt, M., \& Zemp, R. (2013). Microbubbleenhanced ultrasound liberation of mRNA biomarkers in vitro. Ultrasound in Medicine \& Biology, 39(6), 1087-1093. http://dx.doi. org/10.1016/j.ultrasmedbio.2012.12.015. PMid:23562017.

Jia, W. R., Chai, W. M., Tang, L., Wang, Y., Fei, X. C., Han, B. S., \& Chen, M. (2014). Three-dimensional contrast enhanced ultrasound score and dynamic contrast-enhanced magnetic resonance imaging score in evaluating breast tumor angiogenesis: correlation with biological factors. European Journal of Radiology, 83(7), 1098-1105. http://dx.doi.org/10.1016/j.ejrad.2014.03.027. PMid:24794865.

Korn, C., Scholz, B., Hu, J., Srivastava, K., Wojtarowicz, J., Arnsperger, T., Adams, R. H., Boutros, M., Augustin, H. G., \& Augustin, I. (2014). Endothelial cell-derived non-canonical Wnt ligands control vascular pruning in angiogenesis. Development, 141(8), 1757-1766. http://dx.doi.org/10.1242/dev.104422. PMid:24715464.

Lambertini, M., Aftimos, P., Gombos, A., Awada, A., \& Piccart, M. (2018). Breast cancer. In M. A. Dicato \& E. Van Cutsem (Eds.), Side effects of medical cancer therapy: prevention and treatment. Cham: Springer.

Laxmanan, S., Robertson, S. W., Wang, E., Lau, J. S., Briscoe, D. M., \& Mukhopadhyay, D. (2005). Vascular endothelial growth factor impairs the functional ability of dendritic cells through Id pathways. Biochemical and Biophysical Research Communications, 334(1), 193198. http://dx.doi.org/10.1016/j.bbrc.2005.06.065. PMid:16002046.

Lin, Z., Liu, Y., Sun, Y., \& He, X. (2011). Expression of Ets-1, Ang-2 and maspin in ovarian cancer and their role in tumor angiogenesis. Journal of Experimental \& Clinical Cancer Research, 30(1), 31. http://dx.doi.org/10.1186/1756-9966-30-31. PMid:21439064.

Liu, H., Jiang, Y., Dai, Q., Zhu, Q., Wang, L., \& Lu, J. (2014). Peripheral enhancement of breast cancers on contrast-enhanced ultrasound: correlation with microvessel density and vascular endothelial growth factor expression. Ultrasound in Medicine \& Biology, 40(2),
293-299. http://dx.doi.org/10.1016/j.ultrasmedbio.2013.10.004. PMid:24315392.

Liu, Y., Wang, G., Xu, W. G., Song, J. N., Yang, X. Q., \& Wang, W. Y. (2009). Relevance of tumor angiogenesis in occurrence and development of breast cancer. Zhonghua Yi Xue Za Zhi, 89(24), 1687-1689. PMid:19957527.

Mao, J. M., Liu, J., Guo, G., Mao, X. G., \& Li, C. X. (2015). Glioblastoma vasculogenic mimicry: signaling pathways progression and potential anti-angiogenesis targets. Biomarker Research, 3(1), 8. http://dx.doi. org/10.1186/s40364-015-0034-3. PMid:26085929.

Mitra, D., Bhattacharyya, S., Alam, N., Sen, S., Mitra, S., Mandal, S., Vignesh, S., Majumder, B., \& Murmu, N. (2020). Phosphorylation of EphA2 receptor and vasculogenic mimicry is an indicator of poor prognosis in invasive carcinoma of the breast. Breast Cancer Research and Treatment, 179(2), 359-370. PMid:31686261.

Onji, K., Yoshida, S., Tanaka, S., Takemura, Y., Oka, S., Yoshihara, M., Yamada, H., Okajima, M., \& Chayama, K. (2012). Microvascular structure and perfusion imaging of colon cancer by means of ultrasonic contrast. Abdominal Imaging, 37(2), 297-303. http://dx.doi.org/10.1007/s00261-011-9738-5. PMid:21512723.

Piñon, M., Alarcon-Rojo, A., Paniwnyk, L., Mason, T., Luna, L., \& Renteria, A. (2019). Ultrasound for improving the preservation of chicken meat. Food Sci. Technol, 39(Suppl. 1), 129-135. http://dx.doi.org/10.1590/fst.39017.

Saponaro, C., Vagheggini, A., Scarpi, E., Centonze, M., Catacchio, I., Popescu, O., Pastena, M. I., Giotta, F., Silvestris, N., \& Mangia, A. (2018). NHERF1 and tumor microenvironment: a new scene in invasive breast carcinoma. Journal of Experimental \& Clinical Cancer Research, 37(1), 96. http://dx.doi.org/10.1186/s13046-018-0766-7. PMid:29716631.

Shabbir, M. S., Ahmed, H., Maan, A. A., Rehman, A., Afraz, M. T., Iqbal, M. W., Khan, I. M., Amir, R. M., Ashraf, W., Khan, M. R., \& Aadil, R. M. (2020). Effect of non-thermal processing techniques on pathogenic and spoilage microorganisms of milk and milk products. Food Science and Technology. In press. http://dx.doi. org/10.1590/fst.05820.

Sharma, S. G., Aggarwal, N., Gupta, S. D., Singh, M. K., Gupta, R., \& Dinda, A. K. (2011). Angiogenesis in renal cell carcinoma: correlation of microvessel density and microvessel area with other prognostic factors. International Urology and Nephrology, 43(1), 125-129. http://dx.doi.org/10.1007/s11255-010-9779-7. PMid:20535637.

Soysal, S. D., Tzankov, A., \& Muenst, S. E. (2015). Role of the tumor microenvironment in breast cancer. Pathobiology, 82(3-4), 142-152. http://dx.doi.org/10.1159/000430499. PMid:26330355.

Vidal, A. R., Cansian, R. L., Mello, R. O., Kubota, E. H., Demiate, I. M., Zielinski, A. A. F., \& Dornelles, R. C. P. (2020). Effect of ultrasound on the functional and structural properties of hydrolysates of different bovine collagens. Food Sci. Technol, 40(2), 346-353. http://dx.doi.org/10.1590/fst.00319.

Warram, J. M., Sorace, A. G., Saini, R., Borovjagin, A. V., Hoyt, K., \& Zinn, K. R. (2012). Systemic delivery of a breast cancer-detecting adenovirus using targeted microbubbles. Cancer Gene Therapy, 19(8), 545-552. http://dx.doi.org/10.1038/cgt.2012.29. PMid:22653385.

Weidner, N. (1995). Current pathologic methods for measuring intratumoral microvessel density within breast carcinoma and other solid tumors. Breast Cancer Research and Treatment, 36(2), 169-180. http://dx.doi.org/10.1007/BF00666038. PMid:8534865.

Zheng, A., Zhang, L., Song, X., Wang, Y., Wei, M., \& Jin, F. (2019). Clinical implications of a novel prognostic factor AIFM3 in breast cancer patients. BMC Cancer, 19(1), 451. http://dx.doi.org/10.1186/ s12885-019-5659-4. PMid:31088422. 